\title{
Nanotechnology, Drug Delivery Systems and their Potential Applications in Hepatitis B Vaccines
}

\begin{abstract}
Since the Nobel Prize in Physiology or Medicine 1976 Baruch Blumberg and Irving Millman developed the first prophylactic plasma-derived hepatitis B vaccine - also considered the world's first cancer vaccine- so much progress has been made in the development of new immunogenic and safe vaccines. However, improvements are still clearly required since vaccines currently available in the market produce mainly a humoral immune response, require cold storage, a three dose schedule, the costs of the vaccines are high, many developing or low-incoming countries display a poor compliance in vaccination programs, and 5-10\% of the vaccinated individuals are poor or non-responders.

To overcome such drawbacks, nanotechnology has emerged as a new platform for vaccine development. Nanocarrier-based delivery systems offer an opportunity to stimulate both humoral as well as cell-mediated responses and to induce mucosal and systemic immunity simultaneously. Furthermore, nanocarrier-based delivery systems avoid the need for sterile needles. This manuscript reviews the current knowledges on the vailable hepatitis B vaccines and introduces the recent advances in nanocarrier-based hepatitis $B$ vaccine delivery systems. The challenges in the development of needle-free nanotechnologies are also discussed.
\end{abstract}

Keywords: HBV; Prophylactic vaccines; HBsAg; Nanocarrier-based delivery systems; Needle-free nanotechnologies

\begin{abstract}
Mini Review
Volume 1 Issue 2 - 2015

Priscila Perazzo ${ }^{1}$, Nicolás Rodriguez Del Valle $^{1}$, Andrea Sordelli ${ }^{1}$, Rodrigo H Gonzalez ${ }^{2}$, Alejandro D Nusblat ${ }^{2}$ and María L Cuestas ${ }^{1 *}$

${ }^{1}$ Institute of Microbiology and Medical Parasitology, University of Buenos Aires-National Science Research Council (IMPaM, UBA-CONICET), Argentina

${ }^{2}$ Cátedra de Biotecnología y Microbiología Industrial, Facultad de Farmacia y Bioquímica, Universidad de Buenos Aires, Argentina

*Corresponding author: María L Cuestas, Institute of Microbiology and Medical Parasitology, University of Buenos Aires-National Science Research Council (IMPaM, UBACONICET), 2155 Paraguay St, 11th Floor, C1121ABG, Buenos Aires, Argentina, Tel: + 541159509500 ext. 2175; Email: marilucuestas@gmail.com
\end{abstract}

Received: June 20, 2015 | Published: September 11, 2015
Abbreviations: CHB: Chronic Hepatitis B; WHO: World Health Organization; HBV: Hepatitis B Virus; HCC: Hepatocellular Carcinoma; HBsAg: Hepatitis B Surface Antigen; MALT: Mucosa-Associated Lymphoid Tissue; GALT: Gut-Associated Lymphoid Tissue

\section{Introduction}

Chronic hepatitis B (CHB) still remains a major problem of health concern due to its significant morbidity and mortalitiy. According to the World Health Organization (WHO), nearly 240 million people are chronically infected worldwide with hepatitis $B$ virus (HBV) even though the availability of a hepatitis B vaccine placed in the market for more than 30 years [1].

HBV causes almost $50 \%$ of the world's cases of hepatocellular carcinoma (HCC) and about $30 \%$ of all cases of liver cirrhosis [2], leading to over 780,000 annual deaths [3].

Considerable progress has been made in the last 20 years in the construction and development of effective prophylactic hepatitis $B$ vaccines and in the implementation of childhood immunization programs so as to protect future generations. However, several gaps remain to be solved. Firstly, current hepatitis B vaccines induced a systemic immune response with a poor mucosal immunity and require a regimen of at least three intramuscular injections [4-6]. Secondly, the global coverage of a three-dose hepatitis B vaccine is $85-90 \%$, that is, current vaccines have a $10-15 \%$ of poor or non-responders rate [7]. This inadequate HBV vaccination coverage rates, especially in certain high-risk populations represents one of the most important unmet needs in the marketplace. Thirstly, the implementation of hepatitis $B$ vaccination is still considerably low where it is most needed, especially in those low-income countries, such as Central Africa where the prevalence rates of HBV infection are too high $[8,9]$. This issue is secondary to the three dose vaccination schedule, the requirement of cold storage and the availability of sterile needles [10]. Finally, some of the vaccinated infants still sometimes get infected due to vaccine-escape mutants $[11,12]$.

While the cure for CHB still appears a longer term alternative, the immediate and middle term challenges reside in the design of advanced vaccine formulations with improved release features of the immunogen and an improved stimulation of both cellular and humoral immune responses. Progresses have been made in specific areas such as Nanotechnology but this is certainly not enough. Scientists in technological fields in general and nanotechnologies in particular, are tacitly compromised with the challenges standing ahead. The present review describes the main drawbacks of the current available prophylactic hepatitis $B$ vaccines and the state-of-the art of all the nanotechnological approaches reported until now with the aim of improving the host immune response after immunization as well as for optimized and long lasting protection and for overcoming the limiting aspects of these current hepatitis B vaccines.

\section{Discussion}

\section{Hepatitis B vaccines history}

In 1970 , the plasma from an individual chronically infected 
with HBV was inactivated by heat, diluted $1 / 10$ and inoculated in healthy individuals. Thus, this plasma lost the infective but not the immunogenic properties of HBV, causing no disease in the healthy receptors that received it. In addition, these vaccinated receptors turned out to be protected against subsequent challenges with HBV. These purified native forms of the hepatitis B surface antigen (HBsAg) - the primary target of HBV-neutralizing antibodiesconstituted the first evidence that led to the subsequent development of a vaccine obtained by plasmapheresis from human chronic carriers of HBV [13]. In 1981, as a result of the work conducted by Wolf Szmuness, the first vaccine against hepatitis B for widespread human use was licensed in the United States of America. This vaccine is now identified as the "first generation" hepatitis B vaccine. This vaccine has now been replaced by recombinant vaccines that are free of any concerns associated with human blood products [13].

Since 1986, recombinant vaccines produced by genetic engineering have been also licensed as safe and effective against hepatitis B [13].

Both plasma-derived and recombinant vaccines have HBsAg as immunogen and stimulate the active synthesis of anti-HBs antibodies, thus conferring protective humoral immunity in vaccinated individuals. An antibody titer of $\geq 10$ mIU per ml measured 1-3 months after the administration of the last dose of the primary vaccination series is considered a reliable marker of protection against infection [14].

Other potential HBV immunogens, such as pre-S1 and pre-S2 were also evaluated for the development of both prophylactic and therapeutic vaccines. Table 1 shows some of the currently available hepatitis B vaccines around the world.

Regarding recombinant hepatitis B vaccines, two and third generation vaccines were licensed and placed in the market for widespread human use. In second generation vaccines, the HBsAg gene has been inserted into yeast and mammalian cells by means of appropriate expression vectors [15]. Antigen expressed in several genus and species of yeasts, including Saccharomyces cerevisiae (Recombivax-HBTM and Engerix-BTM), Pichia pastoris (Enivac-HB, Shanvac B) and Hansenula polymorpha (ButanNG, GeneVac-B) has been used to produce hepatitis B vaccines for over 20 years [Table 1]. Recombinant HBsAg differs from its native counterpart only in the glycosylation of the protein, which can vary according to the production cell [16].

Table 1: Some of the currently available hepatitis B vaccines around the world.

\begin{tabular}{|c|c|c|}
\hline Name & Type Vaccine & Company \\
\hline Engerix B & Recombinant HBV, HBsAg -2nd generation & GlaxoSmithKline Biologicals \\
\hline ButanNG & Recombinant HBV, HBsAg -2nd generation & Butantan - Brazil \\
\hline GeneVac-B & Recombinant HBV, HBsAg -2nd generation & Serum Institute of India- India \\
\hline Vecon & Recombinant HBV, HBsAg -2nd generation & Biokangtai - China \\
\hline Regevac B & Recombinant HBV, HBsAg -2nd generation & Binnopharm - Russia \\
\hline Enivac-HB & Recombinant HBV, HBsAg -2nd generation & Panacea Biotec-India \\
\hline Shanvac B & Recombinant HBV, HBsAg -2nd generation & Shantha-India \\
\hline Bilive & Hepatitis A and Hepatitis B - Bivalent & Sinovac - China \\
\hline Comvax & $\begin{array}{c}\text { Haemophilus b Conjugate Vaccine (Meningococcal } \\
\text { Conjugate) and Hepatitis B - Bivalent }\end{array}$ & Merck \& Co, Inc \\
\hline Pediarix & $\begin{array}{c}\text { Diphtheria, Tetanus, Pertussis, Hepatitis-B and Hib - } \\
\text { Pentavalent }\end{array}$ & GlaxoSmithKline Biologicals \\
\hline Pentaxim & $\begin{array}{c}\text { Diphtheria, Tetanus, Pertussis, Hepatitis-B and Hib - } \\
\text { Pentavalent }\end{array}$ & Sanofi Pasteur MSD \\
\hline Quinvaxem & $\begin{array}{l}\text { Diphtheria, Tetanus, Pertussis, Hepatitis-B and Hib - } \\
\text { Pentavalent }\end{array}$ & Novartis AG \\
\hline Easyfive-TT & $\begin{array}{c}\text { Diphtheria, Tetanus, Pertussis, Hepatitis-B and Hib - } \\
\text { Pentavalent }\end{array}$ & Panacea Biotec - India \\
\hline HEXYON & $\begin{array}{l}\text { Diphtheria, Tetanus, Pertussis, Hepatitis B, Poliomyelitis, } \\
\text { Haemophilus influenzae type b disease (Hib) - Hexavalent }\end{array}$ & Sanofi Pasteur MSD \\
\hline GenHevacB & HBsAg and PreS2 - 3rd generation & Institut Pasteur-France \\
\hline
\end{tabular}


Only two 3rd generation vaccines are commercially currently available: GenHevacB and Sci-B-Vac. The first one comprises the viral envelope proteins, HBsAg and pre-S2 whereas the remaining one comprises the three HBV envelope proteins: HBsAg, pre-S2 and pre-S1. Both of them are produced in Chinese hamster ovary (CHO) cells. The active component is highly similar to the native counterpart, and it is more potent and immunogenic than the recombinant HBsAg used as immunogen in the existing yeast derived hepatitis $B$ vaccines.

As in the first generation vaccines, the use of 2nd and 3rd generation vaccines in children and young people promotes the seroconversion to $85-90 \%$ of individuals.

\section{Hepatitis B vaccines global market}

The global market for vaccines comprises about $2 \%$ to $3 \%$ of the total pharmaceutical market. Nevertheless, this sector has a high growth rate of $10 \%$ to $15 \%$ per year versus the $5 \%$ to $7 \%$ seen in the overall market. Currently, the global vaccine market has an estimated value of almost $\$ 24$ billion and is projected to rise to $\$ 100$ billion by 2025 [17].

More than 120 new products are in the development pipeline. Half of them - which account for less than $20 \%$ of the global market - are of great importance for the developing countries, since they comprise the $80 \%$ of the worldwide population. The main global vaccine players are Glaxo Smithkline, Sanofi Pasteur, Pfizer, Merck and Novartis. These 5 large multinational corporations make up $80 \%$ of the global market.

In this context the global hepatitis B vaccine market has an estimated value of $\$ 1.02$ billion and will experience a modest growth over the coming years reaching $\$ 1.19$ billion by 2022 , at an annual growth rate of $2.2 \%$ [18].

The eight major markets (US, Canada, France, Germany, Italy, Spain, the UK and Japan) account for more than $55 \%$ of the global market and is estimated to reach more than $62 \%$ in the first years of the next decade (2020).

The moderate growth in HBV vaccine sales will result from the launch of Dynavax Technologies' Heplisav-B and the recently approved (2013) Sanofi Pasteur MSD's Hexyon, with peakyear sales anticipated to reach $\$ 85$ million and $\$ 520$ million, respectively.

Heplisav-B is an adult hepatitis B vaccine in Phase 3 development that utilizes a proprietary TLR9 agonist to elicit a focused immune response providing improved protection against hepatitis B. The ongoing Phase 3 study is designed to provide a sufficiently-sized safety database for the FDA to complete its review of Dynavax's biologics license application.

Hexyon is the first and only fully liquid pediatric hexavalent vaccine, ready-to-use, to protect infants against hepatitis $\mathrm{B}$, diphtheria, tetanus, pertussis, poliomyelitis and invasive infections caused by Haemophilus influenzae type b.

\section{Which are the most common drawbacks of hepatitis B} vaccines?

The stricking non-responders rate(10-15\%) together with the fact that current vaccines require a regimen of at least three intramuscular injections, a guaranteed cold chain from point of manufacture to point of use cold, and the availability of sterile needles, significantly increases the costs and limits the compliance of many low-income countries in the vaccination programs [7]. Furthermore, the currently prophylactic hepatitis B vaccines available in the market produce mainly a systemic immune response with an insignificant mucosal immune response [19]. In this regards, it is widely known that HBV is transmitted by parenteral or permucosal exposure, that is, HBV is considered a mucosal infection. Thus, there is a need to induce a significant long lasting and optimized mucosal immunity.

Prevention of hepatitis B, one of the most prevalent human diseases, requires cheaper and more accessible vaccines. In addition, less invasive strategies and easier storage and transport requirements should also be prioritized. At present, scientists in technological fields in general and nanotechnologies in particular aim to find new technologies that contribute to the accessibility of all kinds of vaccines at lower costs, thus mainly benefiting the underserved populations. Several lines of research are arduously working to generate a new applicable, cheaper and translatable vaccine against hepatitis B using nanotechnology, as it is exposed below. In the following section, we will also gain insight into fundamental aspects related to mucosal immunization using nanotechnology.

\section{Nanodelivery systems for hepatitis B vaccines}

An important issue is the mucosal immunization, an attractive alternative to parenteral vaccines because it offers an opportunity to stimulate both systemic and mucosal immune responses and avoids the need for sterile needles. It is widely known that parenteral vaccines does not usually offer an optimal or long lasting protection against diseases caused by microorganisms that are specially inhaled, ingested or sexually transmitted [19]. Hence, for optimal mucosal protection, induction of immune response via mucosal routes is desirably and thus mucosal vaccines emerge as a new promise and challenge for improving the efficacy of immunization. At present, there are a few mucosal vaccines approved by FDA such as RotaTeq (Merck - Rotavirus vaccine, a live attenuated oral vaccine), Rotarix (GlaxoSmithKline, a live oral attenuated vaccine), Adenovirus type 4 and type 7 vaccine (Teva Pharmaceuticals, a live attenuated oral vaccine) and oral Polio vaccine (GlaxoSmithKline - a live attenuated oral vaccine made from Sabin strains). These are affective because they use live attenuated viruses. It is well known that a prolonged exposure of the immune system to the attenuated agent results in an increased immunogenicity and a long lasting immunity in comparison with traditional injectable counterparts (e.g. oral Polio vaccine versus injectable Polio vaccine, developed by Jonas Salk). However, risk of reversion to virulence makes attenuated vaccines undesirable for immunocompromised individuals and pregnant women. Other mucosal vaccines approved by FDA for human use are the oral typhoid fever vaccine (Vivotif Berna, Crucell Switzerlan LTD, a live attenuated strain of Salmonella typhi -Ty21a-), and the intranasal vaccine against influenza disease (FluMist Quadrivalent, AstraZeneca group, a live attenuated Influenza virus types A and B vaccine). As it is shown, all these mucosal vaccines are made of live attenuated microorganisms. 
Nanotechnology platforms have been employed for newly vaccine developments. It was reported that nanocarrier-based delivery systems may stimulate both humoral and cellular immune responses due to the nanoscale particle size [20]. The nanoparticles do not exceed $1,000 \mathrm{~nm}$, leading this attribution to an easier uptake by phagocytic cells, antigen presenting cells, the mucosa-associated lymphoid tissue or MALT and the gut-associated lymphoid tissue or GALT which secrete the cytokine TGF- $\beta$, which induces IgA secretion [20]. This means, that these nanoparticles facilitates the antigen recognition and presentation, mainly through the specialized epithelial cells known as microfold M cells, which take up and transport antigens across the epithelial membrane to macrophages, dendritic cells and $\mathrm{B}$ and T cells present in the Peyer's patches in the intestinal tract or in the nasal-associated lymphoreticular tissue of the oropharyngeal, the tonsils and adenoids in order to initiate and activate immune respone to the intact antigen [21,22]. Thus $\mathrm{M}$ cells remain good targets for mucosal vaccine-delivery systems. Hence, Nanotechnology is an important tool with the potential of improving or modulating the immunogenicity of a vaccine formulation. It is considered that an ideal nanodelivery system should have the ability to stimulate humoral, cellular and mucosal immune responses [23]. Many nanocarriers have been designed and investigated for their usefulness in the delivery of immunogens and adjuvants to immune cells so as to promote a protective immune response [24,25]. Among them, the most frequently reported as vaccines delivery systems are liposomes, nanoemulsions, nanospheres and polymer-based nanoparticles. The FDA approved a wide range of synthetic and natural polymers for the production of nano/microparticles to prepare controlledrelease delivery systems (Decapeptyl ${ }^{\circledR}$, Lupron Depot ${ }^{\circledR}$, Nutropin Depot $^{\circledR}$, Zoladex $^{\circledR}$, etc).

In this context, and regarding new nanotechnological strategies for improving the efficacy of hepatitis B vaccine, Saraf et al incorporated HBsAg in lipid microparticles for mucosal immunization against hepatitis B [19]. These lipid particles had their own adjuvant effect for immunization via respiratory tract inducing immune responses at both systemic and mucosal sites. For the preparation of these lipid particles containing HBsAg, authors used a double emulsion-solvent evaporation (w/o/w) method. This formulation was stable, immunogenic and cellular uptake was efficiently achieved by systemic and alveolar macrophages. Considerable immune responses were also produced by the developed system due to MALT induction [19]. Makidson et al carried out a pre-clinical evaluation of a novel nanoemulsion-based hepatitis B mucosal vaccine using a nanoemulsion manufactured by emulsification of cetyl pyridinium chloride, Tween $80(5 \%)$ and ethanol (8\%) in water with soybean oil (64\%) using a high speed emulsifier [24]. Results demonstrated that this formulation was safe and effective as a potential hepatitis $B$ vaccine, and that it induced a Th1 associated cellular immunity to adequately control viral replication, a potential benefit as a therapeutic vaccine for CHB patients [26].
Jaganathan \& Vyas [27] developed surface-modified DLlactide/glycolide copolymer (PLGA) microspheres with chitosan for nasal immunization using recombinant HBsAg [27]. These modified PLGA microspheres (cationic microspheres) produced humoral (both systemic and mucosal) and cellular immune responses upon nasal administration [27].

PLGA nanoparticles were also used for loading HBsAg, stabilizing this immunogen by co-encapsulation of trehalose and $\mathrm{Mg}(\mathrm{OH})_{2}$. The Ulex europaeus 1 lectin was anchored on the surface of these nanoparticles to target them to M-cells of the intestinal peyer's patches as a strategy for oral immunization against hepatitis B [26]. Authors proposed that these lectinized nanoparticles could be a promising carrier-adjuvant for the targeted oral-mucosal immunization [28]. Other strategies to prevent degradation and increase the absorption of administered antigen, is its inclusion into biodegradable microspheres or liposomes or their expression in bacterial or viral vectors and plants $[29,30]$.

Delivery systems containing monophosphoril lipid A (MLP) combined with aluminum salts or the saponin adjuvant Quil A have been developed and demonstrated to enhance both humoral and TH1 immune responses [23]. Calcium phosphate nanoparticles generated by mixing and stirring calcium chloride, sodium phosphate and sodium citrate were used in clinical trials, and demonstrated to be safe and to have an increased or even similar immune response to that achieved using aluminum salts [23]. They also displayed a mucosal immunity protection [23].

Another interesting work, showed that for the intranasal delivery of HBsAg, recombinant haemagglutinin (HA) protein from the Influenza virus was complexed with liposomes loaded with HBsAg. HA offered a better adherence of the immunogen to the mucosal surface than pristine liposomes [31]. When systemic and mucosal antigen titers were measured after immunization, results demonstrated that anti-HBsAg IgG titers were higher in HA-liposomes than in the pristine counterparts, but lower than alum vaccines administered intramuscularly. Secretory IgA in mucosal fluids were measured after 6 weeks of immunization showing higher titers using the liposomal complexes, especially those recovered on the surface with the HA protein, whereas in the case of the alum-HBsAg administered intramuscularly, titers rendered not legible. Through the measurement of the cytokines profile, it was observed that the Th1 response was dominant when the formulation HA-liposomes were administrated intranasally [31].

Tafaghodi et al used trimethylated chitosan and chitosan nanoparticles loaded with HBsAg for nasal immunizaton in a murine model [32] and results demonstrated that these nanoparticles may be promising for mucosal immunization. Chitosan is a natural copolymer with the ability to induce both humoral and cellular responses and even of producing Il-2 and 
IFN-Y, indicative of a Th1 response. Borges et al, used a similar immunization system with HBsAg encapsulated into alginatecoated chitosan nanoparticles, and similar results they achieved. Authors also reported that the cellular responses they measured were accompanied with anti-HBsAg.specific IgG in the sera and IgA in the intestinal mucosa [33].

A novel adaptated version of the liposome/niosome system is the development of bilosomes, which are non-ionic surfactant vesicles-based systems with bile salts. This nanotechnological platform displayed a high stability after oral administration of an immunogen, and also induces significant antibody titers. These formulations are so stable, that the need for "cold chain" supply is no necessary [21].

In summary, the expected advancements obtained using all these nanotechnological platforms are administration regimes less frequent, more compliant vaccination programs, lower costs, optimal mucosal protection, an enhanced humoral and cellular immune response and less stringent cold requirement due to a higher stability [34]. These issues are highly relevant mainly in low-incoming countries with high HBV endemicity. Moreover, these achievements may extend their applicability to other vaccines.

\section{Conclusion}

Nanotechnology has contributed to improve the administration and delivery of many drugs and immunogens. A press release by Cientifica Ltd. foresees a critical expansion in the nano-based drug delivery market from its current $\$ 3.4$ billion (10\% of the total drug delivery market) to about $\$ 220$ billion in 2015 . Regardless the apparently difficult implementation, a number of reasons support the fact that nanotechnologies can provide unique solutions also in poorer societies, such as many areas of Africa, Asia and South America:

i. Strong nanotechnology research in developing countries oriented to solve specific prophylactic and therapeutic problems of their own communities will make these new technologies more affordable. Programs like the Horizon 2020 make possible this kind of initiatives.

ii. Increasing funding by public and private organizations for research on specific diseases affecting primarily the developing world; e.g. the Global Alliance for Vaccine and Immunization (the GAVI Alliance) and the Hepatitis B Foundation together with the Centers for Disease Control and Prevention, the American Liver Foundation and the Pennsylvania Department of Health among other partnerships is stimulating research on everything related to hepatitis B and additional funding is being devoted.

iii. Nanotechnology-related mucosal immunization may improve the prophylactic success by stimulating both humoral and cell-mediated responses, inducing mucosal and systemic immunity simultaneously, doing administration regimes less fre- quent, vaccination strategies more compliant and needle free and attaining higher responding rates and altogether reducing substantially the vaccine regimens costs. Moreover, they can enhance the effectiveness of approved vaccines and extend their applicability to other ones by providing means to overcome the current drawbacks, such as high costs, the requirement for cold storage and the availability of sterile needles.

iv. Commitment between pharmaceutical companies and governments, international agencies to make prices of new vaccines and technologies more affordable.

Finally, to design and implement nanotechnologies at reasonable costs and to make them affordable to people in developing countries are key ethical and scientific challenges. This would probably make possible transforming a certainly chronic and even fatal disease into a highly preventable one, for all the patients regardless their socioeconomic status. This is a main endeavour of many researchers and the main message of the present work.

\section{Acknowledgement}

This work was partially supported by the University of Buenos Aires (Grant UBACyT 20020130200222BA). Nusblat A and Cuestas ML are members of CONICET.

\section{References}

1. Hepatitis B (2015). WHO: World Health Organization Fact sheet $\mathrm{N}^{\circ} 204$.

2. El-Seraq HB (2011) Hepatocellular carcinoma. N Engl J Med 365(12): 1118-1127.

3. Gish RG, Locarnini S (2007) Genotyping and genomic sequencing in clinical practice. Clin Liver Dis 11(4): 761-795.

4. Vajdy M, O’Hagan DT (2001) Microparticles for intranasal immunization. Adv Drug Deliv Rev 51(1-3): 127-141.

5. Davis SS (2001) Nasal vaccines. Adv Drug Deliv Rev 51(1-3): 21-42.

6. Illum E, Davies SS (2001) Nasal vaccination: a non-invasive vaccine delivery method that holds great promise for the future. Adv Drug Deliv Rev 511-3: 1-3.

7. Komatsu H (2014) Hepatitis B virus: where do we stand and what is the next step for eradication? World J Gastroenterol 20(27): 89989016.

8. Komas NP, Vickos U, Hübschen JM, Béré A, Manirakiza A, et al (2013) Cross-sectional study of hepatitis B virus infection in rural communities, Central African Republic. BMC Infect Dis 13: 286.

9. Ducancelle A, Abguguen P, Birguel J, Mansour W, Pivert A, et al (2013) High endemicity and low molecular diversity of hepatitis B virus infections in pregnant women in a rural district of North Cameroon. PLoS One 8(11): e80346.

10. Giudice EL, Campbell JD (2006) Needle-free vaccine delivery. Adv Drug Deliv Rev 58(1): 68-69.

11. Mathet VL, Cuestas ML, Ruiz V, Minassian ML, Rivero C, et al. (2006) Detection of hepatitis B virus (HBV) genotype E carried -even in the 
presence of high titters of anti-HBs antibodies-by an Argentinean patient of African descent who had received vaccination against HBV. J Clin Microbiol 44(9): 3435-3439.

12. Lin YM, Jow GM, Mu SC, Chen BF (2013) Naturally occurring hepatitis $B$ virus B-cell and T-cell epitope mutants in hepatitis B vaccinated children. Scientific World Journal 2013: 571875.

13. Mathet VL, Cuestas ML, Oubiña JR (2014) Virus hepatitis B. Chapter 24. In: Virología Médica, Corpus ed, Argentina.

14. Yu AS, Cheung RC, Keeffe EB (2006) Hepatitis B vaccines. Infect Dis Clin North Am 20(1): 27-45.

15. Cregg J, Tschopp J, Stillman C (1987) High-Level Expression and efficient assembly of hepatitis B surface antigen in the methylotrophic yeast Pichia pastoris. Nature 5: 479-485.

16. Kim MW, Rhee SK, Kim JY, Shimma Y, Chiba Y, et al (2004) Characterization of $\mathrm{N}$-linked oligosaccharides assembled on secretory recombinant glucose oxidase and cell wall mannoproteins from the methylotrophic yeast Hansenula polymorpha. Glycobiology 14(3): 243-251.

17. Kaddar M (2013) Global vaccine market features and trends. WHO report.

18. PharmaPoint: Prophylactic Hepatitis B Virus Vaccines - Global Drug Forecast and Market Analysis to 2022.

19. Saraf S, Mishra D, Asthana A, Jain R, Singh S, et al (2006) Lipid microparticles for mucosal immunization against hepatitis $B$. Vaccine 24(1): 45-56

20. Kim MG, Park JY, Kim G, Shim G, Oh YK (2014) Nanotechnology and vaccine development. Asian J Pharm Sci 9(5): 227-235.

21. Wilkhu J, McNeil SE, Kirby DJ, Perrie Y (2011) Formualtion design considerations for oral vaccines. Ther Deliv 2(9): 1141-1164.

22. Van Ginkel FW, Nguyen HH, McGhee JR (2000) Vaccines for mucosal immunity to combat emerging infectious diseases. Emerg Infect Dis 6(2): 123-132.

23. Peek LJ, Russell M, Berkland C (2008) Nanotechnology in vaccine delivery. Adv Drug Deliv Rev 60(8): 915-928.
24. Demento SL, Cui W, Criscione JM, Stern E, Tulipan J, et al. (2012) Role of sustained antigen release from nanoparticle vaccines in shaping the T cell memory phenotype. Biomaterials 33(19): 4957-4964.

25. Gregory AE, Titball R, Williamson D (2013) Vaccine delivery using nanoparticles. Front Cell Infect Microbiol 3: 13.

26. Makison PE, Bielinska AU, Nigavekar SS, Janczak KW, Knowlton J, et al (2008) Pre-clinical evaluation of a novel nanoemulsion-based hepatitis B mucosal vaccine. PLoS ONE 3(8): e2954.

27. Jaganathan KS, Vyas SP (2006) Strong systemic and mucosal immune responses to surface-modified PLGA microspheres containing recombinant hepatitis $B$ antigen administered intranasally. Vaccine 24(19): 4201-4211.

28. Gupta PN, Khatri K, Goyal AK, Mishra N, Vyas SP (2007) M-cell targeted biodegradable PLGA nanoparticles for oral immunization against hepatitis B. J Drug Target 15(10): 701-713.

29. Mestecky J, Moldoveanu Z, Michalek SM, Morrow CD, Compans RW, et al (1997) Current options for vaccine delivery systems by mucosal routes. J Control Release 48(2-3): 243-257.

30. Pniewski T (2012) Is an oral plant-based vaccine against hepatitis B virus possible? Curr Pharm Biotechnol 13(15): 2692-2704.

31. Tiwari S, Verma SK, Agrawal GP, Vyas SP (2011) Viral Protein Complexed Liposomes for Intranasal Delivery of Hepatitis B Surface Antigen. Int J Pharm 413(1-2): 211-219.

32. Tafaghodi M, Saluja V, Kersten GF, Kraan H, Slütter B, et al (2012) Hepatitis B Surface Antigen Nanoparticles Coated with Chitosan and Trimethyl Chitosan: Impact of Formulation on Physicochemical and Immunological Characteristics. Vaccine 30(36): 5341-5348.

33. Borges O, Tavares J, de Sousa A, Borchard G, Junginger HE, et al (2007) Evaluation of the immune response following a short oral vaccination schedule with hepatitis B antigen encapsulated into alginate-coated chitosan nanoparticles. Eur J Pharm Sci 32(4-5): 278-290.

34. Carra JH, Martins KA, Schokman RD, Robinson CG, Steffens JT, et al. (2015) A thermostable, chromatographically purified Ebola nanoVLP vaccine. J Transl Med 13: 228. 